\title{
Bacillus subtilis mutants harbouring a single copy of the rRNA operon exhibit severe defects in growth and sporulation
}

Correspondence

Fujio Kawamura

kawamura@rikkyo.ne.jp

Received 5 October 2009

Revised 28 June 2010

Accepted 14 July 2010

\author{
Hideaki Nanamiya,† Makiko Sato, Kenta Masuda, Mikiko Sato, \\ Tetsuya Wada, Shota Suzuki, Yousuke Natori, Masato Katano, \\ Genki Akanumał and Fujio Kawamura
}

Laboratory of Molecular Genetics and Research Information Center for Extremophile, College of Science, Rikkyo University, Toshima-ku Nishi-ikebukuro 3-34-1, Tokyo 171-8501, Japan

\begin{abstract}
The number of copies of rRNA genes in bacterial genomes differs greatly among bacterial species. It is difficult to determine the functional significance of the heterogeneity of each rRNA operon fully due to the existence of multiple rRNA operons and because the sequence heterogeneity among the rRNA genes is extremely low. To overcome this problem, we sequentially deleted the ten rrn operons of Bacillus subtilis and constructed seven mutant strains that each harboured a single $r r n$ operon (either $r r n A, B, D, E, I, J$ or $O$ ) in their genome. The growth rates and sporulation frequencies of these mutants were reduced drastically compared with those of the wild-type strain, and this was probably due to decreased levels of ribosomes in the mutants. Interestingly, the ability to sporulate varied significantly among the mutant strains. These mutants have proved to be invaluable in our initial attempts to reveal the functional significance of the heterogeneity of each rRNA operon.
\end{abstract}

\section{INTRODUCTION}

Most rRNA ( $\mathrm{rrn}$ ) operons in bacterial cells are composed of three rRNA genes, which encode 16S, $23 \mathrm{~S}$ and 5S rRNA in that order (Klappenbach et al., 2000). The copy number of rRNA operons differs among many bacterial genomes, although most of the genes that encode ribosomal proteins are present as a single copy (Klappenbach et al., 2001). For example, Mycoplasma genitalium, a pathogenic bacterium whose genome size is very small (580 $074 \mathrm{bp}$ ) (Fraser et al., 1995), contains only one rRNA operon in its genome, whereas the genomes of Escherichia coli and Bacillus subtilis contain seven and ten $r r n$ operons, respectively (Klappenbach et al., 2001). In general, organisms that possess multiple $r r n$ operons are considered to be able to grow faster than those that possess one or two operons. However, it is possible to delete one rRNA operon in either E. coli or B. subtilis without having a major effect on cell growth rate or physiology (Ellwood \& Nomura, 1980; Widom et al., 1988), which suggests that the full number of

tPresent address: Cell-free Science and Technology Research Center, Ehime University, Bunkyo-cho, Matsuyama 790-8577, Japan.

łPresent address: Department of Biotechnology, Graduate School of Agriculture and Life Sciences, University of Tokyo, Bunkyo-ku, Tokyo 113-8657, Japan.

Supplementary methods describing the construction of the strains and a supplementary table showing the oligonucleotide primers used in this study are available with the online version of this paper. copies of the rRNA operon is not required for rapid growth in these two organisms. Instead, it is assumed that many prokaryotes evolved multiple rRNA operons to cope with a variety of environmental conditions (Condon et al., 1992, 1995). This raises the possibility that a functional differentiation exists among multiple rRNA operons. However, it is difficult to elucidate the functional significance of the heterogeneity among these operons because, in general, this heterogeneity is limited and the rRNA operons are highly conserved (Nomura, 1999).

B. subtilis, which is the best-characterized Gram-positive bacterium, has been studied extensively as a model for unicellular differentiation because of its ability to form dormant spores (Schaeffer et al., 1965; Hoch, 1993; Grossman, 1995). Ten rrn operons ( $r r n O, A, J, W, I, H$, $G, E, D, B)$, which include the $r r n J-r r n W$ and $r r n I-r r n H-$ $r r n G$ clusters, have been identified in the genome of $B$. subtilis (Widom et al., 1988; Kunst et al., 1997; Henkin, 2002) (Fig. 1a). Clusters of tRNA genes are located downstream of the $5 S$ rRNA genes in $r r n J, I, E, D$ and $B$, upstream of the 16S rRNA gene in $r r n E$, and in the spacer regions between the 16S rRNA and 23S rRNA genes in rrnO and rrnA (Fig. 2). To our knowledge, there have been few studies that have focused on the heterogeneity of rRNA sequences among the ten $r r n$ operons in B. subtilis and the functional significance of this heterogenity. Therefore, we sequentially deleted the rrn operons in B. subtilis strain 168 and constructed seven mutant strains, each of which 
(a)

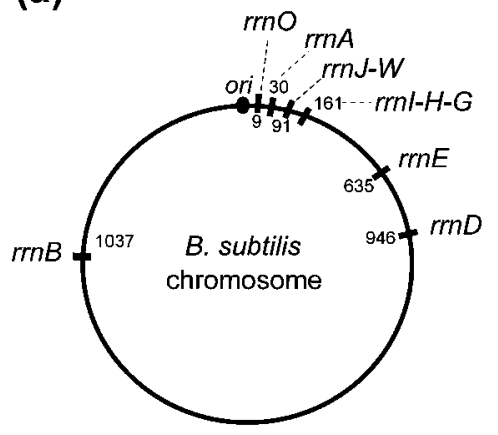

(c)

\section{S rRNA}

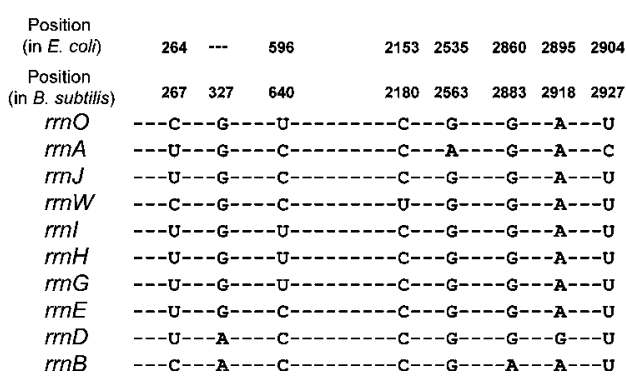

(b)

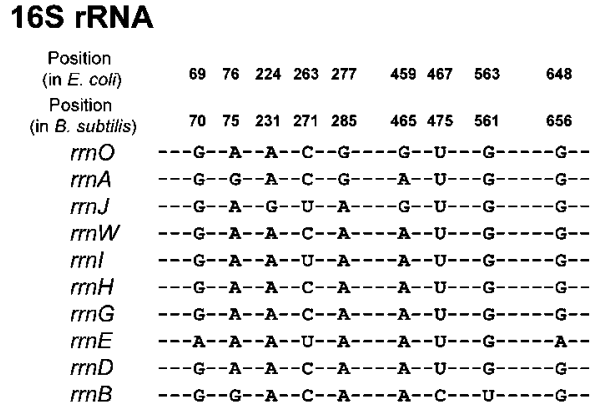

(d)

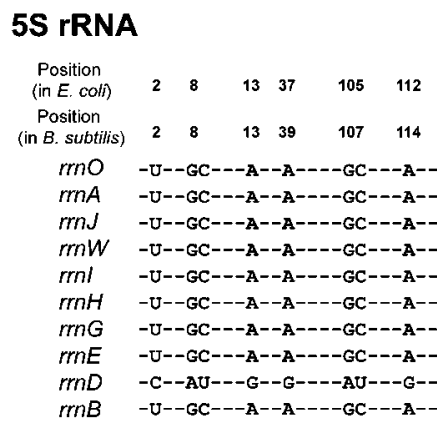

Fig. 1. (a) Location of the rrn operons in Bacillus subtilis. The numbers shown at the position of each $r r n$ operon indicate the distances (kbp) between the origin of replication and the bases that correspond to the $5^{\prime}$ end of the mature 16S rRNA in $r r n O, A, J, I, D$, $E$ and $B$. Heterogeneity of $16 S$ (b), $23 S$ (c), and $5 S$ rRNA (d) sequences between the different $r r n$ operons in $B$. subtilis. The numbers shown above the alignments are the positions (in both $B$. subtilis and $E$. coli numbering) at which differences were found between the rRNAs; the $5^{\prime}$ ends of mature 16S, 23S and 5S rRNA genes were each designated 1. harboured a single $r r n$ operon (either $\operatorname{rrn} A, B, D, E, I, J$ or $O)$ in their genome. In this study, the growth profiles, ribosome content and sporulation frequency of the seven mutant strains were monitored to examine whether or not the function of each individual rRNA operon was equivalent.

\section{METHODS}

Strains and media. All the B. subtilis strains used in this study were isogenic with B. subtilis strain 168 and are listed in Table 1. The details of their construction are available in the supplementary material available with the online version of this paper. The media used included LB and LB agar (Sambrook \& Russell, 2001), CI and CII media (Ashikaga et al., 2000), minimal glucose (MG) agar (Rutberg, 1969) supplemented with $0.05 \%$ amicase (Sigma) (MG-CH agar), and $2 \times$ Schaeffer's sporulation medium supplemented with $0.1 \%$ glucose $(2 \times$ SG; Leighton \& Doi, 1971). When required, antibiotics were added at the following concentrations: $5 \mu \mathrm{g}$ chloramphenicol $\mathrm{ml}^{-1}, 0.5 \mu \mathrm{g}$ erythromycin $\mathrm{ml}^{-1}$ and $5 \mu \mathrm{g}$ kanamycin $\mathrm{ml}^{-1}$.

Southern blot hybridization analysis. One microgram of chromosomal DNA from each of the wild-type and mutant strains was cut with Bst1107I (SnaI; Takara) and then the digested DNA was electrophoresed through a $0.8 \%$ agarose gel in $1 \times$ TAE buffer (Sambrook \& Russell, 2001). After the gel had been soaked in $0.25 \mathrm{M}$ $\mathrm{HCl}$ for $5 \mathrm{~min}$ with gentle shaking, it was soaked twice in denaturing buffer $(0.5 \mathrm{M} \mathrm{NaOH}, 1.5 \mathrm{M} \mathrm{NaCl})$ for $15 \mathrm{~min}$ and twice in neutralization buffer $(0.5 \mathrm{M}$ Tris $/ \mathrm{HCl} \mathrm{pH} 7.5,3 \mathrm{M} \mathrm{NaCl})$ for 15 min. Next, the digested DNA was blotted onto a Hybond- ${ }^{+}$ membrane (GE Healthcare) by capillary transfer. After the membrane had been baked at $120{ }^{\circ} \mathrm{C}$ for $30 \mathrm{~min}$, it was soaked in hybridization buffer [ $5 \times$ saline sodium citrate (SSC), $50 \%$ deionized formamide, $0.02 \%$ SDS, $0.1 \% \mathrm{~N}$-lauroylsarcosine and $2 \%$ Blocking Reagent (Roche Diagnostics)] that contained a 23S-specific RNA probe at a final concentration of $100 \mathrm{ng} \mathrm{ml}^{-1}$, and incubated at $40{ }^{\circ} \mathrm{C}$ for $16 \mathrm{~h}$. Binding of the probe was detected using NBT/BCIP as the substrate for alkaline phosphatase according to the manufacturer's protocol (Roche Diagnostics). The template for the $23 \mathrm{~S}$ probe was prepared from B. subtilis strain 168 by PCR using the primers rrnPro.F and rrnPro.R (Supplementary Table S1).

Assay for sporulation. B. subtilis cells were grown in $2 \times \mathrm{SG}$ medium for $24 \mathrm{~h}$ at $37{ }^{\circ} \mathrm{C}$ with shaking, and heat-resistant spores were counted by heating the cells at $80{ }^{\circ} \mathrm{C}$ for $10 \mathrm{~min}$ and then plating them on LB agar plates.

Microscopic imaging. Cells were grown in $2 \times$ SG medium in the presence of $5 \mu \mathrm{g} \mathrm{ml}^{-1}$ of FM4-64 (Invitrogen) for $20-22 \mathrm{~h}$ at $37{ }^{\circ} \mathrm{C}$ with shaking. A $0.1 \mathrm{ml}$ portion of the culture was centrifuged at $12000 \mathrm{~g}$ for $1 \mathrm{~min}$ at $25^{\circ} \mathrm{C}$. The cell pellet was resuspended in $30 \mu \mathrm{l}$ of the culture supernatant and mounted on microscope slides covered with a thin film of $1 \%$ agarose in distilled water. All images were acquired with a SenSys-1401E air-cooled CCD camera (Roper Scientific) attached to an Olympus BX50 microscope equipped with an $100 \times$ UPlanApo objective.

Sucrose density gradient sedimentation analysis. Cells were grown in LB medium at $37{ }^{\circ} \mathrm{C}$ with shaking to early exponential phase $\left(\mathrm{OD}_{600} \sim 0.2\right)$, harvested and then disrupted by passing them through a French press (Aminco) at 55.2 MPa. The cell debris was then removed by centrifugation as described previously (Natori et al., 2007) and the supernatants were used as crude cell extracts. The $\mathrm{OD}_{600}$ of each sample was measured before (designated $D_{\mathrm{b}}$ ) and after (designated $D_{\mathrm{a}}$ ) passage through the French press, and the efficiency of cell lysis (designated $E$ ) for each sample was calculated from the following equation: $E=D_{\mathrm{a}} / D_{\mathrm{b}}$. 


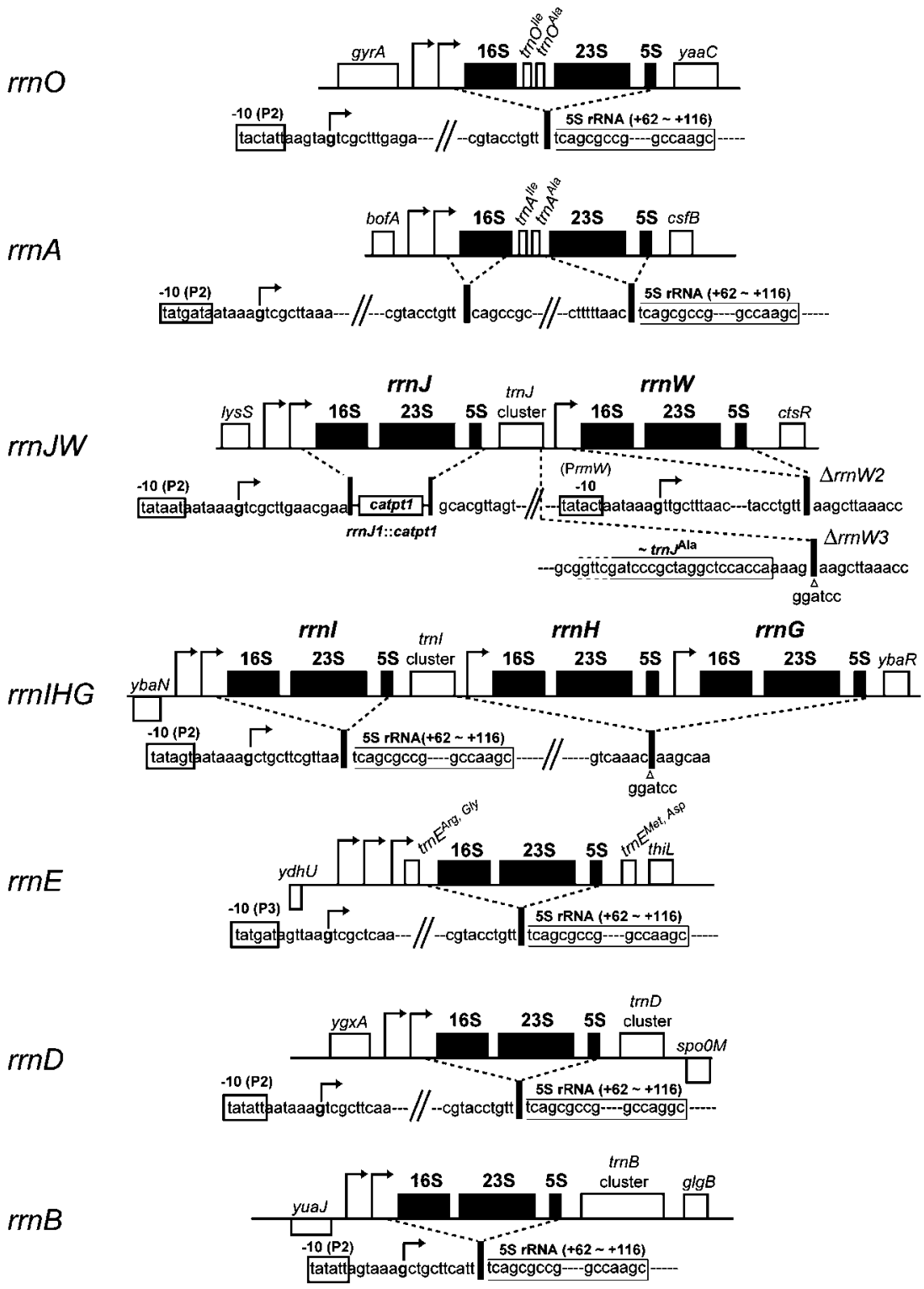

Fig. 2. Sequence alignment of seven $B$. subtilis mutants, each of which contained the $\operatorname{rnO}, A, J, I, E, D$, or $B$ operon. Detailed protocols for the construction of these mutants are described in the supplementary material available with the online version of this paper. rRNA genes and tRNA genes are shown by closed and open boxes, respectively. The -10 regions of the promoters and the transcriptional start sites (indicated in boldface) are shown by rectangles and bars with arrows, respectively. The junction points created by the introduction of the deletion are shown by the bars connected to dotted lines, and the dotted lines indicate the regions that were deleted. The additional restriction sites created by the introduction of the deletion mutation of $r \mathrm{rHG}$, as well as $r r n W 3$, at the junction points, are shown with an open arrowhead. The 5S rRNA sequences that remained in each deletion mutant are boxed.
The OD units (designated $U$ ) of each sample that had been prepared from $500 \mathrm{ml}$ of $\mathrm{LB}$ medium were calculated as $U=500 \times L \times E$, where $L$ is the $\mathrm{OD}_{600}$ value for each culture at harvesting. Next, the $A_{260}$ of the extract from the wild-type cells was measured and the volume of wild-type extract (designated $A_{\mathrm{wt}}$ ) that contained $10 A_{260}$ units was calculated. This corresponded to the volume of extract used for the

Table 1. B. subtilis strains used in this study

\begin{tabular}{|c|c|c|}
\hline Strain & Genotype (characteristics) & Source or reference \\
\hline 168 & $\operatorname{trp} C 2$ (wild-type) & Laboratory stock \\
\hline RIK539 & $\operatorname{trpC2} \Delta r r n H G 1 \Delta r r n O 1 \Delta r r n D 1 \Delta r r n E 1 \Delta r r n B 2 \Delta r r n I 2 \Delta r r n W 2 \Delta r r n J 1:: c a t\left(\Delta 9 r r n A^{+}\right)$ & This study \\
\hline RIK540 & $\operatorname{trpC2} \Delta r r n H G 1 \Delta r r n O 1 \Delta r r n D 1 \Delta r r n E 1 \quad \Delta r r n A 1 \Delta r r n I 2 \Delta r r n W 2 \Delta r r n J 1:: c a t\left(\Delta 9 r r n B^{+}\right)$ & This study \\
\hline RIK541 & $\operatorname{trpC2} \Delta r r n H G 1 \Delta r r n O 1 \Delta r r n E 1 \Delta r r n B 2 \Delta r r n A 1 \Delta r r n I 2 \Delta r r n W 2 \Delta r r n J 1:: c a t\left(\Delta 9 r r n D^{+}\right)$ & This study \\
\hline RIK542 & $\operatorname{trpC2} \Delta r r n H G 1 \Delta r r n O 1 \Delta r r n D 1 \Delta r r n E 1 \Delta r r n B 2 \Delta r r n A 1 \Delta r r n W 2 \Delta r r n J 1:: c a t\left(\Delta 9 r r n I^{+}\right)$ & This study \\
\hline RIK543 & $\operatorname{trpC2} \Delta r r n H G 1 \Delta r r n D 1 \Delta r r n E 1 \Delta r r n B 2 \Delta r r n A 1 \Delta r r n I 2 \Delta r r n W 2 \Delta r r n J 1:: c a t\left(\Delta 9 r r n O^{+}\right)$ & This study \\
\hline RIK545 & $\operatorname{trpC2} \Delta r r n H G 1 \Delta r r n O 1 \Delta r r n D 1 \Delta r r n B 2 \Delta r r n A 1 \Delta r r n I 2 \Delta r r n W 2 \Delta r r n J 1:: c a t\left(\Delta 9 r r n E^{+}\right)$ & This study \\
\hline RIK551 & $\operatorname{trpC2} \Delta r r n H G 1 \Delta r r n O 1 \Delta r r n D 1 \Delta r r n E 1 \Delta r r n B 2 \Delta r r n A 1 \Delta r r n I 2 \Delta r r n W 3\left(\Delta 9 r r n J^{+}\right)$ & This study \\
\hline
\end{tabular}


sucrose density gradient sedimentation analysis. Then, the appropriate volume of extract for each mutant (designated $\mathrm{p} A_{r r n X}$ ) for sucrose density gradient sedimentation analysis was determined according to the following equation: $\mathrm{p} A_{r r n X}=A_{\mathrm{wt}} \times\left(U_{\mathrm{wt}} / U_{r r n X}\right)$, where $\mathrm{U}_{\mathrm{wt}}$ and $\mathrm{U}_{r r n X}$ are the OD units of the wild-type sample and each mutant sample, respectively.

To normalize the applied volumes of the samples further, each strain was cultured in $1 \mathrm{ml} \mathrm{LB}$ medium at $37^{\circ} \mathrm{C}$ with shaking and harvested at an $\mathrm{OD}_{600}$ of $\sim 0.2$. Chromosomal DNA was then extracted from these samples. The amounts of chromosomal DNA extracted from the cells were quantified by spectrophotometric assay and agarose gel electrophoresis. Then the ratio of DNA content of each mutant strain to that of the wild-type (designated $R$ ) was calculated using the following equation, which normalized the values with respect to the ratio of $\mathrm{OD}_{600}$ values for the mutant and wildtype cultures: $R_{r r n X}=\left(\mathrm{OD}_{\mathrm{wt}} / \mathrm{OD}_{r r n X}\right)\left(C_{\mathrm{wt}} / C_{r r n X}\right)$, where $\mathrm{OD}_{\mathrm{wt}}$ and $\mathrm{OD}_{r r n X}$ are the $\mathrm{OD}_{600}$ values for the wild-type and mutant cultures, respectively, and $C_{\mathrm{wt}}$ and $C_{r r n X}$ are the total amounts of chromosomal DNA extracted from the wild-type and mutant cells, respectively. Finally, the volume of extract for each mutant (designated $A_{r r n X}$ ) to be used for sucrose density gradient sedimentation analysis was determined from the following equation: $A_{r r n X}=\mathrm{p} A_{r r n X} \times R_{r r n X}$.

The aliquots of extract were layered onto $10-40 \%$ sucrose density gradients and centrifuged at $4{ }^{\circ} \mathrm{C}$ for $17.5 \mathrm{~h}$ at $65000 \mathrm{~g}$ (HITACHI $\mathrm{P} 40 \mathrm{ST}$ rotor). Absorbance profiles were monitored at $254 \mathrm{~nm}$ using a Piston Gradient Fractionator (Biocomp) and a Bio-mini UV Monitor (ATTO).

\section{RESULTS}

\section{Construction of seven mutant strains harbouring a single rrn operon (rrnA, $B, D, E, I, J$ or $O)$}

Before we sequentially deleted the rRNA operons in the genome of $B$. subtilis strain 168 , we resequenced all ten rrn operons. The complete genome sequence of $B$. subtilis was obtained originally using strain 168 , which is used as the parental strain in our laboratory. We identified sequence heterogeneity at nine, eight and eight locations in the 16S, 23S and 5S rRNA genes, respectively (Fig. 1b, c, d). It was noteworthy that, of the ten $5 S$ genes, only the $5 \mathrm{~S}$ gene in the $r r n D$ operon differed substantially from the other $5 \mathrm{~S}$ genes. This suggested that the $5 \mathrm{~S}$ rRNA expressed from the $r r n D$ operon might have a different role in the function of ribosome to $5 \mathrm{~S}$ rRNAs encoded by the other operons.

To determine whether the function of all the rRNA operons was equivalent, we decided to construct a range of mutant strains, each of which carried only one of the rRNA operons in their genome. Some laboratory strains of $B$. subtilis contain only nine $r r n$ operons due to spontaneous deletions within $r r n W, H$ or $G$ (Widom et al., 1988). This indicates that these operons are dispensable for growth in B. subtilis in the presence of the other rrn genes. Therefore, we focused on the other seven rrn operons, i.e. the first operons of the contiguous $r r n J-r r n W$ and $r r n I-r r n H-r r n G$ clusters ( $r r n J$ and $r r n I)$, and the noncontiguous operons $r r n O, A, E, D$ and $B$. We constructed seven mutant strains that each harboured a single $r r n$ operon (either $\operatorname{rrn} A, B, D$, $E, I, J$ or $O$ ) in their genome (Fig. 2).

Multiple deletions of $r r n$ operons have been constructed previously in E. coli by the sequential deletion of the $16 \mathrm{~S}$ and $23 \mathrm{~S}$ rRNA genes; however, the 5S rRNA genes were left intact (Asai et al., 1999a, b). On the other hand, as described above, it is assumed that 5S rRNA expressed from the $r r n D$ operon in $B$. subtilis has a different role in the function of ribosome to $5 \mathrm{~S}$ rRNA encoded by the other operons (Fig. 1d). In order to investigate the function of $5 \mathrm{~S}$ rRNA expressed from $r r n D$, it was desirable to construct a strain which carries multiple deletion mutations of 16S, $23 \mathrm{~S}$ and 5S rRNA genes. Therefore, we attempted to delete the $5 \mathrm{~S}$ rRNA sequences in each $\mathrm{rrn}$ operon. To obtain the PCR products, which were used as donor DNAs to delete the rRNA operons, the upstream and downstream regions of each rRNA operon were first amplified by PCR. The two fragments obtained were then used simultaneously as the template for the next PCR amplification to generate the DNA fragments covering the deletion of the rRNA, as described in the supplementary material. During these processes, we used the forward primer rrnXDF (Supplementary Table S1), which was designed to be used commonly to amplify the downstream regions of each rRNA operon, to cover the bases between +62 and $+84\left(+1\right.$ denotes the $5^{\prime}$ end of the mature $5 \mathrm{~S}$ rRNA) of each $5 S$ rRNA gene. Therefore, the $3^{\prime}$ portion of the 5S rRNA gene (bases +62 to +116 ) was included in the deletion mutants of $r r n O, I, E, D$ and $B$ (Fig. 2). In addition, given that $\operatorname{rrnADF}$, which was used for amplifying the downstream region of $r r n A$, was designed in the same manner as rrnXDF, the deletion mutant of rrnA also contained the $3^{\prime}$ portion of the 5S rRNA gene (Fig. 2). On the other hand, the deletion mutants of $r r n W$ ( $\Delta r r n W 3), G$ and $H$ did not contain any portion of the $5 \mathrm{~S}$ rRNA gene (Fig. 2), even though a BamHI site (ggatcc), which was created via the cloning procedures as described in the supplementary material, was included in each deletion (Fig. 2). In addition, the deletion mutant of $\mathrm{rrnW}$ $(\Delta r r W 2)$, as well as the replacement of $r r n J$ with the cat gene (rrnJ1::catpt1), did not contain any portion of the 5S rRNA gene (Fig. 2).

There are various tRNA genes in each operon (Fig. 2). It has not been clarified, so far, whether these tRNA genes are actually required for cell proliferation. Therefore, most of these tRNA genes, including the trnJ, trnI, trnE, trnD and $\operatorname{trn} B$ clusters, remained intact after the introduction of the deletion mutations into the rRNA operons (Fig. 2). On the other hand, two tRNA genes, tRNA ${ }^{\text {Ile }}$ and tRNA ${ }^{\text {Ala }}$, are located between the $16 \mathrm{~S}$ and $23 \mathrm{~S}$ rRNA genes in both the rrnO and rrnA operons (Loughney et al., 1982; Ogasawara et al., 1983). Given that the DNA sequences of these two genes are the same, the tRNA genes in the rrnA operon were left intact, but the equivalent tRNA genes in $r r n O$ were deleted, as shown in Fig. 2. In addition, given that many of these tRNA genes are co-expressed from the 
promoters of each rRNA operon, none of the rrn promoters was deleted.

The $\Delta 9 r r J^{+}$(RIK551) mutant, which harboured a single $r r n J$ operon, contained the $\Delta r r n W 3$ mutation, whereas the other mutants that harboured a single rRNA operon contained the $\Delta r r n W 2$ mutation (Fig. 2, Table 1). During the construction of the $\Delta 9 \mathrm{rrnJ}^{+}$strain, transformants that carried a tandem duplication of $r r n J$ were obtained. It is likely that this duplication of the rrnJ operon could be generated by unequal crossing-over via the promoter region of $\mathrm{rrnW}$ (from bases -166 to $-50 ;+1$ denotes the $5^{\prime}$ end of the mature $16 \mathrm{~S}$ rRNA of $\mathrm{rrnW}$ ), which was present in the $\Delta r r n W 2$ deletion mutant. Therefore, we deleted the entire $r r n W$ operon, which included the region between $-166 \mathrm{nt}$ from the $5^{\prime}$ end of the mature $16 \mathrm{~S} \mathrm{rRNA}$ and the $3^{\prime}$ end of the mature 5S rRNA in $r r n W$, and named this deletion $\Delta r r n W 3$ (Fig. 2).

The detailed protocols for the construction of the mutant that carried multiple deletions of the rrn operons are described in the supplementary material. The deletion of the rRNA genes in these strains was verified by Southern blot analysis using a riboprobe that was specific for the $23 \mathrm{~S}$ rRNA gene. This confirmed that each strain contained a single $r r n$ operon in its genome; a typical result is shown in Fig. 3.

\section{Growth rates and intracellular ribosome levels differ among the mutant strains}

First we monitored the growth profiles of the seven mutant strains, which each harboured only one rRNA operon, in a nutrient-rich medium. All of the strains that carried a single rRNA operon grew more slowly than the wild-type strain. This result is consistent with a previous report in which the introduction of multiple deletions of rRNA

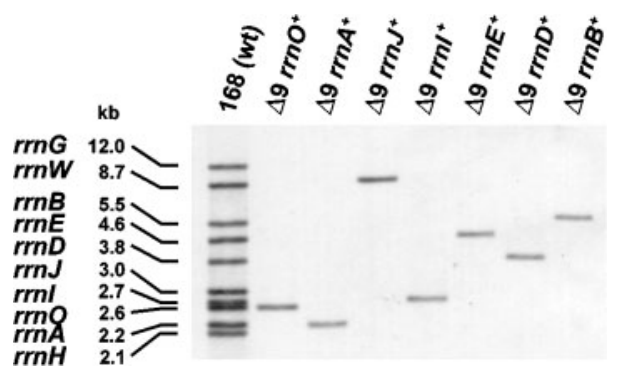

Fig. 3. Confirmation of the multiple deletion of the $r r n$ operons by Southern blot analysis. Bst1107l digests were electrophoresed through a $0.8 \%$ agarose gel and probed with a 23S-specific RNA probe as described in Methods. Given that the Bst1107I site located between trnJ and rrnW was deleted simultaneously with the sequence of $r r n W$, the size of the band derived from the $r r n J$ 23S rRNA in the strain $\Delta 9 \mathrm{rrnJ}^{+}$(approx. $9.6 \mathrm{~kb}$ ) differed from that of the wild-type (approx. $3.0 \mathrm{~kb}$ ). operons in E. coli caused a decrease in the growth rate (Asai et al., 1999b). However, the growth rates of the B. subtilis mutants differed: the doubling times of the strains that harboured $r r n O\left(\Delta 9 r r n O^{+}\right), r r n A\left(\Delta 9 r r n A^{+}\right), r r n J(\Delta 9$ $\left.r r n J^{+}\right), \quad r r n I\left(\Delta 9 r r n I^{+}\right), \quad r r n E\left(\Delta 9 r r n E^{+}\right), \quad r r n D(\Delta 9$ $\left.r r n D^{+}\right)$and $r r n B\left(\Delta 9 r r n B^{+}\right)$were $69 \mathrm{~min}, 58 \mathrm{~min}$, $57 \mathrm{~min}, 66 \mathrm{~min}, 62 \mathrm{~min}, 79 \mathrm{~min}$ and $80 \mathrm{~min}$, respectively, whereas that of the wild-type strain was 23 min (Fig. 4). One plausible explanation for these observations is that the expression of rRNA and/or the intracellular ribosome concentration is decreased significantly in the $\Delta 9 \mathrm{rrnD}{ }^{+}$ and $\Delta 9 \mathrm{rrnB}^{+}$strains. To test this hypothesis, the intracellular concentration of $70 \mathrm{~S}$ ribosomes was monitored by sucrose density gradient sedimentation analysis. The volume of each sample that was applied to the gradient was calculated by determining the ratio of chromosomal DNA between wild-type and mutant cells as described in Methods. Each of the strains that harboured a single rRNA operon contained a lower level of $70 \mathrm{~S}$ ribosomes than that found in the wild-type cells (Fig. 5). The relative levels of $70 \mathrm{~S}$ ribosomes in the $\Delta 9 r r n I^{+}, \Delta 9 r r n E^{+}, \Delta 9 r r n D^{+}$and $\Delta 9 \mathrm{rrnB}^{+}$strains were $0.28,0.3,0.20$ and 0.23 , respectively (where the level of 70S ribosomes in the wild-type cells was set at 1.0), whereas in the $\Delta 9 \mathrm{rrnO}^{+}, \Delta 9 \mathrm{rrnA}^{+}$and $\Delta 9$ $r r n J^{+}$strains the levels were $0.34,0.29$ and 0.35 , respectively (Fig. 5). It was assumed that, during the exponential growth phase, the level of ribosomes in each mutant that harboured a single rRNA operon was, at least in part, dependent on the location of each operon in the genome. Namely, as the distance between the origin of DNA replication and the location of the rRNA operon in the genome increased, the level of $70 \mathrm{~S}$ ribosomes decreased (Figs 1a and 5). Given that multiple replication forks of the chromosomal DNA are found in exponentially growing cells, the copy numbers of $r r n O, r r n A$ and $r r n J$, which are
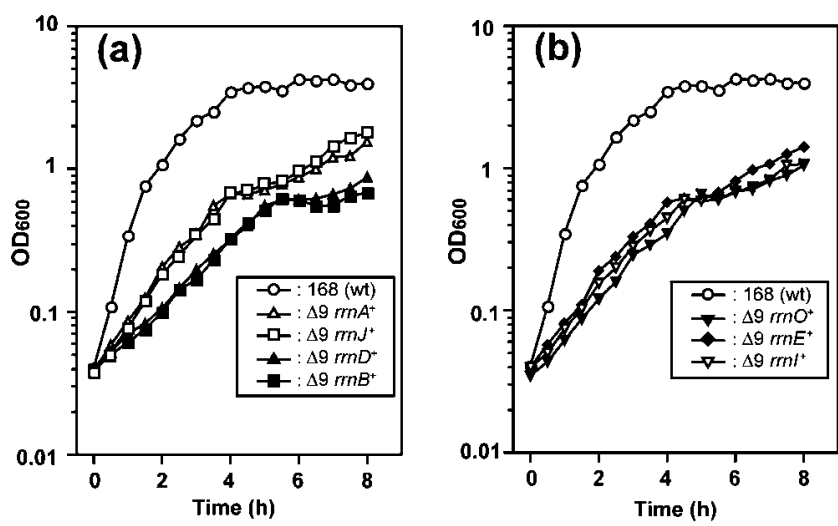

Fig. 4. $(a, b)$ Growth characteristics of various $\Delta 9 r r n$ strains that harboured a single rRNA operon. Cells were incubated in LB medium at $37{ }^{\circ} \mathrm{C}$ with shaking and the $\mathrm{OD}_{600}$ of the cultures was measured at the indicated times after inoculation. The curves for the wild-type strain are shown in both (a) and (b). 


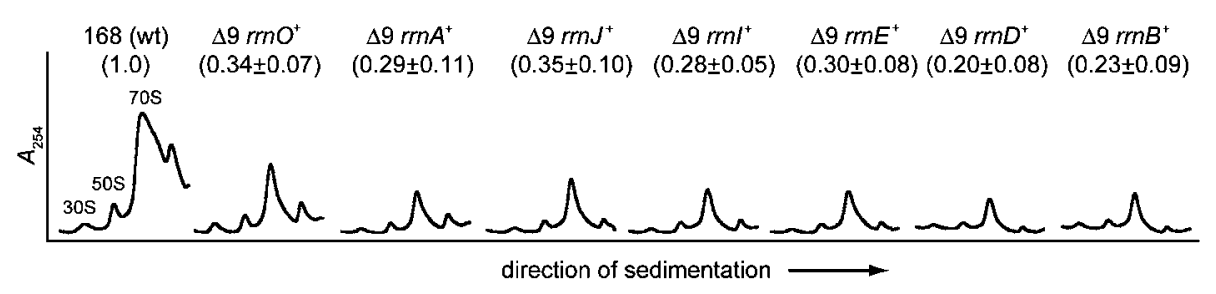

Fig. 5. Ribosome sedimentation profiles from the strains that harboured a single rRNA operon. Crude cell-extracts were sedimented through a 10-40\% sucrose gradient as described in Methods. Representative results of the ribosome profiles obtained by three independent experiments are shown. The areas of the $70 \mathrm{~S}$ ribosome peaks were measured for each strain. The values shown in parentheses are relative to the value for the $70 S$ peak in wild-type cells, which was set at 1.0. The values are the means $\pm S D$ of three independent experiments.

located near the origin of replication, would be relatively higher than those of $r r n D$ and $r r n B$, which are located approximately $950 \mathrm{~kb}$ and $1000 \mathrm{~kb}$, respectively, from the origin of replication. Therefore, the expression of rRNA genes in $\Delta 9 r r n O^{+}, \Delta 9 r r n A^{+}$and $\Delta 9 \mathrm{rrnJ}^{+}$would be expected to be higher than in $\Delta 9 r r n D^{+}$and $\Delta 9 r r n B^{+}$, thereby resulting in larger amounts of ribosomes in $\Delta 9$ $r r n \mathrm{O}^{+}, \Delta 9 \mathrm{rrnA}^{+}$and $\Delta 9 r r n J^{+}$cells as compared with those in $\Delta 9 \mathrm{rrnD}{ }^{+}$and $\Delta 9 \mathrm{rrnB}^{+}$cells. Together with the observation that the growth rates of $\Delta 9 \mathrm{rrnD}^{+}$and $\Delta 9$ $r r n B^{+}$were significantly lower than those of $\Delta 9 r r A^{+}$and $\Delta 9 \mathrm{rrnJ}^{+}$(Fig. 4a), these results suggest that the slower growth rates observed in the strains that harboured a single rRNA operon might be due to a reduction in the intracellular levels of ribosomes.

\section{Sporulation frequencies differ among the strains that harbour a single rRNA operon}

The sporulation frequencies of the strains containing a single rRNA operon were determined as described in Methods. Among the mutant strains, $\Delta 9 \mathrm{rrnE}^{+}$yielded the highest number of spores $\left(1.7 \times 10^{6} \mathrm{ml}^{-1}\right)$ at $24 \mathrm{~h}$ after inoculation in $2 \times$ Schaeffer's sporulation medium (Table 2). However, this number was two orders of magnitude lower than that obtained from the wild-type strain $\left(6.8 \times 10^{8} \mathrm{ml}^{-1}\right)$ (Table 2). In addition, the numbers of spores obtained from $\Delta 9 \mathrm{rrnI}^{+}$and $\Delta 9 \mathrm{rrnB}^{+}\left(3.1 \times 10^{3}\right.$ $\mathrm{ml}^{-1}$ and $6.5 \times 10^{2} \mathrm{ml}^{-1}$, respectively) were five and six orders of magnitude lower than that obtained for the wildtype (Table 2). Moreover, the number of spores produced by the $\Delta 9 r r n D^{+}$strain was below the detection limit (Table 2). Therefore, it is clear from these results that sporulation efficiency differed significantly among the mutant strains. To determine the stage of sporulation that is blocked in the mutant strains harbouring a single $r r$ operon we next carried out fluorescence microscopy analysis using the vital membrane stain FM 4-64. When cells were incubated in $2 \times$ Schaeffer's sporulation medium at $37{ }^{\circ} \mathrm{C}$ for $20-22 \mathrm{~h}$, most of the wild-type cells were observed as free spores (Fig. 6a). However, the mutant cells, except for $\Delta 9 r r E^{+}$cells (Fig. 6b), were not able to form spores and were blocked at the early stages of sporulation, where the apparent asymmetrical septum, seen in wild-type cells, was not observed (Fig. 6c, d show a representative result obtained with $\Delta 9 \mathrm{rrnB}^{+}$cells). These results strongly suggested that a single rRNA operon was not sufficient to support efficient spore development. On the other hand, refractile bodies were observed in $\Delta 9$ $r r n E^{+}$, although the number was apparently low when compared with that of the wild-type strain (Fig. 6b). This result was coincident with the fact that $\Delta 9 \mathrm{rrnE}^{+}$exhibited the highest number of spores among the mutant strains (Table 2).

Next we examined the efficiency of the mutant strains to develop the ability to take up exogenous DNA. The induction of the developmental pathways for competence was delayed in all of the strains that contained a single rRNA operon (data not shown). This could be a result of the slow-growth phenotypes. Given that all of the mutant strains were able to develop competence, although with a lower frequency than the wild-type strain, these strains should be very useful for genetic analyses and for investigating the functions of each rRNA gene.

Table 2. Sporulation of the strains harbouring a single rRNA operon

\begin{tabular}{|lccc|}
\hline \multirow{2}{*}{ Strain } & \multicolumn{2}{c}{ C.f.u. $\mathbf{m l}^{-\mathbf{1}^{*}}$} & \multirow{2}{*}{ Frequency $(\%)^{\star}$} \\
\cline { 2 - 3 } & Total & Spores & \\
\hline 168 (wild-type) & $8.1 \times 10^{8}$ & $6.8 \times 10^{8}$ & $84.0 \pm 3.4$ \\
RIK543 $\left(\Delta 9 \mathrm{rrnO}^{+}\right)$ & $7.6 \times 10^{8}$ & $4.4 \times 10^{4}$ & $5.6( \pm 2.5) \times 10^{-3}$ \\
RIK539 $\left(\Delta 9 \mathrm{rrnA}^{+}\right)$ & $5.1 \times 10^{8}$ & $5.6 \times 10^{3}$ & $10( \pm 5.4) \times 10^{-4}$ \\
RIK551 $\left(\Delta 9 \mathrm{rrnJ}^{+}\right)$ & $6.9 \times 10^{8}$ & $5.5 \times 10^{3}$ & $6.5( \pm 4.7) \times 10^{-4}$ \\
RIK542 $\left(\Delta 9 \mathrm{rrnI}^{+}\right)$ & $4.9 \times 10^{8}$ & $3.1 \times 10^{3}$ & $5.8( \pm 3.4) \times 10^{-4}$ \\
RIK545 $\left(\Delta 9 \mathrm{rrnE}^{+}\right)$ & $6.7 \times 10^{8}$ & $1.7 \times 10^{6}$ & $2.5( \pm 2.2) \times 10^{-1}$ \\
RIK541 $\left(\Delta 9 \mathrm{rrnD}^{+}\right)$ & $4.1 \times 10^{8}$ & $<10$ & $<0.000003$ \\
RIK540 $\left(\Delta 9 \mathrm{rrnB}^{+}\right)$ & $5.2 \times 10^{8}$ & $6.5 \times 10^{2}$ & $1.4( \pm 1.1) \times 10^{-4}$ \\
& & & \\
\hline
\end{tabular}

${ }^{\star}$ Means of three independent experiments $( \pm \mathrm{SD}$ for sporulation frequency). 
(a)

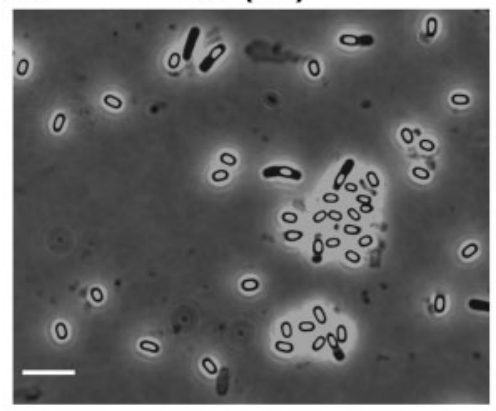

(c) $\Delta 9 \mathrm{rrnB}^{+}$(Phase contrast)

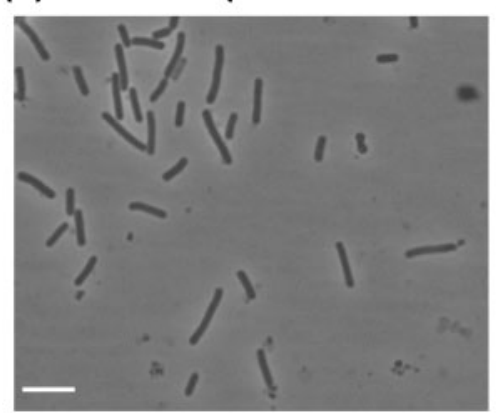

(b) $\Delta 9 r n E^{+}$

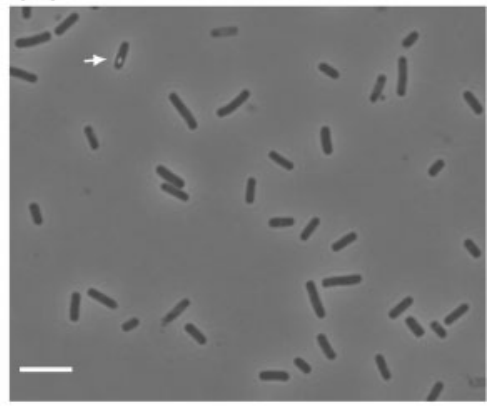

(d) $\Delta 9 \mathrm{rrnB}^{+}$(FM4-64)

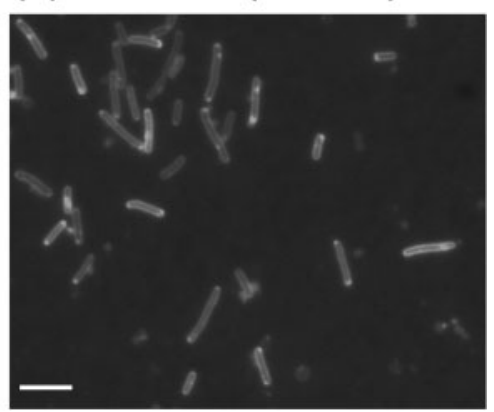

Fig. 6. Microscopic observation of wild-type (a), $\Delta 9 r r n E^{+}$(b), and $\Delta 9 r r n B^{+}$(c, d)cells. Cells grown in $2 \times$ Schaeffer's sporulation medium at $37{ }^{\circ} \mathrm{C}$ for $20-22 \mathrm{~h}$ were collected and phase-contrast images $(\mathrm{a}, \mathrm{b}, \mathrm{c})$ and $\mathrm{a}$ fluorescence image using FM4-64 (d) were obtained. A refractile body found in $\Delta 9 \mathrm{rrnE}^{+}$ (b) is indicated by an arrow. Bars, $5 \mu \mathrm{m}$.

\section{DISCUSSION}

An E. coli strain that lacks all seven $r r n$ operons has been constructed previously (Asai et al., 1999a, b). In this strain, which carried no chromosomal copy of an intact rRNA operon, rRNA molecules were expressed from a multicopy plasmid that contained a single rRNA operon. This strain has contributed to the identification and characterization of novel mutations in the rRNA genes that confer resistance to antibiotics (Recht \& Puglisi, 2001). It has also contributed to the characterization of the functional domain in the rRNA molecules that is required for translation (Sergiev et al., 2005; Hirabayashi et al., 2006). Similar genetic systems have also been developed using Synechococcus PCC 7942 (Monshupanee et al., 2006) and Mycobacterium smegmatis (Sander et al., 1996; Hobbie et al., 2006). However, at least to our knowledge, there have been no other reports of studies that clarify the functional differentiation among multiple rRNA operons, with the exception of a study in Plasmodium, in which two distinct genes that encode 18S rRNA were shown to be expressed differentially in different stages of the life cycle (Gunderson et al., 1987). Therefore, we constructed seven different novel strains of $B$. subtilis, each of which harboured a different single rRNA operon.

One notable difference among the strains that harboured a single rRNA operon was with respect to spore formation. All of the mutant strains grew more slowly than the wildtype during the exponential growth phase (Fig. 4). In addition, we found that the expression of spoOA from the Ps promoter, which is recognized by the sporulation initiation sigma factor, $\sigma^{\mathrm{H}}$ (Chibazakura et al., 1991;
Predich et al., 1992), was hardly detected in the $\Delta 9 \mathrm{rrnO}^{+}$ strain (data not shown). Also, microscopic observations revealed that most of the mutant cells were blocked at the early stages of sporulation, where no obvious asymmetrical septum was found (Fig. 6). These results strongly suggested that the reduced sporulation frequencies seen in the mutant strains were caused by a delay in the entry into the initiation of sporulation, which could be due to the reduced growth rates caused by a decrease in the intracellular levels of ribosomes during the exponential growth phase (Fig. 5).

On the other hand, sporulation frequency differed considerably among these strains (Table 2). It was noteworthy that the sporulation frequencies of $\Delta 9 \mathrm{rrnD}^{+}$ and $\Delta 9 \mathrm{rrnB}^{+}$were extremely low when compared with that of the other mutant strains (Table 2). The difference in spore formation might also be explained by the heterogeneity of the rRNA sequences. For example, in the $23 \mathrm{~S}$ rRNA expressed from eight of the rrn operons in B. subtilis, the nucleotide at position 327 (in B. subtilis numbering) is G. However, in the $23 \mathrm{~S}$ rRNA expressed from $r r n D$ or $r r n B$ this nucleotide is replaced by A (Fig. 1). Nucleotide 327 is located within a $39 \mathrm{nt}$ sequence that is inserted near helix 16-18 in domain I of the B. subtilis $23 \mathrm{~S}$ rRNA but is not present in the E. coli sequence. This additional sequence is commonly found in Bacillus species, which suggests that this region could play an important role in sporulation. Although the molecular details remain unclear, the G327A substitution found in $r r n D$ and $r r n B$ might result in a reduction in the numbers of spores produced. However, it could not simply be concluded that the variation in sporulation ability among these mutants was due to the 
heterogeneity among the rRNA genes. Rather, as described above, growth rates, as well as intracellular levels of ribosomes, in each mutant during exponential growth would strongly affect the initiation of spore formation. In fact, the intracellular levels of ribosomes in strains $\Delta 9$ $r r n D^{+}$and $\Delta 9 r r n B^{+}$were particularly low among the mutant strains that harboured a single rRNA operon, and the doubling times of $\Delta 9 \mathrm{rrnD}^{+}$and $\Delta 9 \mathrm{rrnB}^{+}$were relatively longer than those of the other mutant strains during exponential growth (Figs 4 and 5). It is thus strongly suggested that a drastic reduction in the sporulation frequency of the $\Delta 9 \mathrm{rrnD}^{+}$and $\Delta 9 \mathrm{rrnB}^{+}$is due to severe growth defects during the exponential growth phase.

In conclusion, the deletion mutants that we have constructed have proved invaluable in the initial attempts described herein to clarify the functional significance of each rRNA operon. However, at present, we cannot fully explain the significance of the chromosomal location of each rRNA operon using these strains. To resolve this issue, mutant strains that harbour a single rRNA operon should be constructed in which each individual rRNA operon is introduced into the same chromosomal location and transcribed from the same promoter. Given that the molecular genetic system in B. subtilis has been well established due to the ability of the bacterium to develop natural genetic competence, these seven strains, which each harbour a single rRNA operon, should provide novel insights into the significance of the presence of multiple rRNA operons in bacteria in the near future.

\section{ACKNOWLEDGEMENTS}

We are grateful to Roy H. Doi for helpful discussions and critical reading of the manuscript. This work was supported by the Frontier Project 'Adaptation and Evolution of Extremophile', and in part by the Ministry of Education, Culture, Sports, Science, and Technology of Japan [a grant-in-aid for scientific research (C) (F. K.), and by a grant-in-aid for creative scientific research (16GS0304) (F.K.)]. In addition, this work was partly supported by a research grant from the Noda Institute for Scientific Research (NISR) to F. K.

\section{REFERENCES}

Asai, T., Zaporojets, D., Squires, C. \& Squires, C. L. (1999a). An Escherichia coli strain with all chromosomal rRNA operons inactivated: complete exchange of rRNA genes between bacteria. Proc Natl Acad Sci U S A 96, 1971-1976.

Asai, T., Condon, C., Voulgaris, J., Zaporojets, D., Shen, B., Al-omar, M., Squires, C. \& Squires, C. L. (1999b). Construction and initial characterization of Escherichia coli strains with few or no intact chromosomal rRNA operons. J Bacteriol 181, 3803-3809.

Ashikaga, S., Nanamiya, H., Ohashi, Y. \& Kawamura, F. (2000). Natural genetic competence in Bacillus subtilis Natto OK2. J Bacteriol 182, 2411-2415

Chibazakura, T., Kawamura, F. \& Takahashi, H. (1991). Differential regulation of spoOA transcription in Bacillus subtilis: glucose represses promoter switching at the initiation of sporulation. J Bacteriol 173, 2625-2632.
Condon, C., Philips, J., Fu, Z.-Y., Squires, C. \& Squires, C. L. (1992). Comparison of the expression of the seven ribosomal RNA operons in Escherichia coli. EMBO J 11, 4175-4185.

Condon, C., Liveris, D., Squires, C., Schwartz, I. \& Squires, C. L. (1995). rRNA operon multiplicity in Escherichia coli and the physiological implications of rrn inactivation. J Bacteriol 177, 41524156.

Ellwood, M. \& Nomura, M. (1980). Deletion of a ribosomal ribonucleic acid operon in Escherichia coli. J Bacteriol 143, 1077-1080.

Fraser, C. M., Gocayne, J. D., White, O., Adams, M. D., Clayton, R. A., Fleischmann, R. D., Bult, C. J., Kerlavage, A. R., Sutton, G. \& other authors (1995). The minimal gene complement of Mycoplasma genitalium. Science 270, 397-403.

Grossman, A. D. (1995). Genetic networks controlling the initiation of sporulation and the development of genetic competence in Bacillus subtilis. Annu Rev Genet 29, 477-508.

Gunderson, J. H., Sogin, M. L., Wollett, G., Hollingdale, M., de la Cruz, V. F., Waters, A. P. \& McCutchan, T. F. (1987). Structurally distinct, stage-specific ribosomes occur in Plasmodium. Science 238, 933-937.

Henkin, T. M. (2002). Ribosomes, protein synthesis factors, and tRNA synthetases. In Bacillus subtilis and its Closest Relatives: from Genes to Cells, pp. 313-322. Edited by A. L. Sonenshein, J. A. Hoch \& R. Losick. Washington, DC: American Society for Microbiology.

Hirabayashi, N., Sato, N. S. \& Suzuki, T. (2006). Conserved loop sequence of helix 69 in Escherichia coli 23S rRNA is involved in A-site tRNA binding and translational fidelity. J Biol Chem 281, 1720317211.

Hobbie, S. N., Bruell, C., Kalapala, S., Akshay, S., Schmidt, S., Pfister, P. \& Böttger, E. C. (2006). A genetic model to investigate drug-target interactions at the ribosomal decoding site. Biochimie 88, 1033-1043.

Hoch, J. A. (1993). Regulation of the phosphorelay and the initiation of sporulation in Bacillus subtilis. Annu Rev Microbiol 47, 441-465.

Klappenbach, J. A., Dunbar, J. M. \& Schmidt, T. M. (2000). rRNA operon copy number reflects ecological strategies of bacteria. Appl Environ Microbiol 66, 1328-1333.

Klappenbach, J. A., Saxman, P. R., Cole, J. R. \& Schmidt, T. M. (2001). rrndb: the ribosomal RNA operon copy number database. Nucleic Acids Res 29, 181-184.

Kunst, F., Ogasawara, N., Moszer, I., Albertini, A. M., Alloni, G., Azevedo, V., Bertero, M. G., Bessieres, P., Bolotin, A. \& other authors (1997). The complete genome sequence of the Gram-positive bacterium Bacillus subtilis. Nature 390, 249-256.

Leighton, T. J. \& Doi, R. H. (1971). The stability of messenger ribonucleic acid during sporulation in Bacillus subtilis. J Biol Chem 246, 3189-3195.

Loughney, K., Lund, E. \& Dahlberg, J. E. (1982). tRNA genes are found between the 16S and 23S rRNA genes in Bacillus subtilis. Nucleic Acids Res 10, 1607-1624.

Monshupanee, T., Fa-aroonsawat, S. \& Chungjatupornchai, W. (2006). A cyanobacterial strain with all chromosomal rRNA operons inactivated: a single nucleotide mutation of $23 \mathrm{~S}$ rRNA confers temperature-sensitive phenotypes. Microbiology 152, 1417-1425.

Natori, Y., Nanamiya, H., Akanuma, G., Kosono, S., Kudo, T., Ochi, K. \& Kawamura, F. (2007). A fail-safe system for the ribosome under zinc-limiting conditions in Bacillus subtilis. Mol Microbiol 63, 294307.

Nomura, M. (1999). Engineering of bacterial ribosomes: replacement of all seven Escherichia coli rRNA operons by a single plasmidencoded operon. Proc Natl Acad Sci U S A 96, 1820-1822. 
Ogasawara, N., Moriya, S. \& Yoshikawa, H. (1983). Structure and organization of rRNA operons in the region of the replication origin of the Bacillus subtilis chromosome. Nucleic Acids Res 11, 6301-6318.

Predich, M., Nair, G. \& Smith, I. (1992). Bacillus subtilis early sporulation genes kinA, spoOF, and spoOA are transcribed by the RNA polymerase containing $\sigma^{\mathrm{H}}$. J Bacteriol 174, 2771-2778.

Recht, M. I. \& Puglisi, J. D. (2001). Aminoglycoside resistance with homogeneous and heterogeneous populations of antibiotic-resistant ribosomes. Antimicrob Agents Chemother 45, 2414-2419.

Rutberg, L. (1969). Mapping a temperate bacteriophage active on Bacillus subtilis. J Virol 3, 38-44.

Sambrook, J. \& Russell, D. (2001). Molecular Cloning: a Laboratory Manual, 3rd edn. Cold Spring Harbor, NY: Cold Spring Harbor Laboratory.
Sander, P., Prammananan, T. \& Böttger, E. C. (1996). Introducing mutations into a chromosomal rRNA gene using a genetically modified eubacterial host with a single rRNA operon. Mol Microbiol 22, 841-848.

Schaeffer, P., Millet, J. \& Aubert, J. (1965). Catabolic repression of bacterial sporulation. Proc Natl Acad Sci U S A 54, 704-711.

Sergiev, P. V., Lesnyak, D. V., Kiparisov, S. V., Burakovsky, D. E., Leonov, A. A., Bogdanov, A. A., Brimacombe, R. \& Dontsova, O. A. (2005). Function of the ribosomal E-site: a mutagenesis study. Nucleic Acids Res 33, 6048-6056.

Widom, R. L., Jarvis, E. D., LaFauci, G. \& Rudner, R. (1988). Instability of rRNA operons in Bacillus subtilis. J Bacteriol 170, 605-610.

Edited by: S. J. Foster 